\title{
Structured Perceptual Arrays and the Modulation of Fitts's Law: Examining Saccadic Eye Movements
}

\author{
Naseem Al-Aidroos \\ Department of Psychology \\ University of Toronto, Ontario, Canada \\ Martin H. Fischer \\ Department of Psychology \\ University of Dundee, Scotland
}

\author{
Jos J. Adam \\ Department of Movement Sciences \\ University of Maastricht, The Netherlands \\ Jay Pratt \\ Department of Psychology \\ University of Toronto, Ontario, Canada
}

\begin{abstract}
On the basis of recent observations of a modulation of Fitts's law for manual pointing movements in structured visual arrays (J. J. Adam, R. Mol, J. Pratt, \& M. H. Fischer, 2006; J. Pratt, J. J. Adam, \& M. H. Fischer, 2007), the authors examined whether a similar modulation occurs for saccadic eye movements. Healthy participants $(N=19)$ made horizontal saccades to targets that appeared randomly in 1 of 4 positions, either on an empty background or within 1 of 4 placeholder boxes. Whereas in previous studies, placeholders caused a decrease in movement time (MT) without the normal decrease in movement accuracy predicted by Fitts's law, placeholders in the present experiment increased saccadic accuracy (decreased endpoint variability) without an increase in MT. The present results extend the findings of J. J. Adam et al. of a modulation of Fitts's law from the temporal domain to the spatial domain and from manual movements to eye movements.
\end{abstract}

Keywords: motor performance, saccadic eye movements, visual perception, visuomotor

\footnotetext{
A $\mathrm{s}$ a rapid aimed movement increases in amplitude, the time to make the movement increases or the accuracy of the movement must decrease. This fundamental relationship between amplitude (distance), speed, and accuracy was reported more than 100 years ago (Woodworth, 1899) and has since been algebraically formalized (Fitts, 1954; Fitts \& Peterson, 1964), resulting in one of the relatively few laws in human behavior: Fitts's law (for a review, see Plamondon \& Alimi, 1997). Recently, however, investigators have noted deviations from this relationship when people make manual reaching movements in the presence of competing, but irrelevant, visual stimuli, such as placeholders (Adam, Mol, Pratt, \& Fischer, 2006; Pratt, Adam, \& Fischer, 2007). In the present study, we investigated whether such placeholders would have a similar effect on saccades (the bal-
}

listic eye movements that people use to reorient gaze) to further delineate the cause of the deviations.

In their initial investigation of the effect of visual placeholders on motor control, Adam et al. (2006) recorded reaching movements from a starting point to targets that appeared suddenly at one of a number of locations arranged linearly on the axis of movement (see Figure 1 for a similar experimental setup). On half of the trials, an array of placeholders marked all the potential target locations; on the other half, no placeholders were present. Because the size of the targets did not vary, the required accuracy of the movements stayed constant. Thus, in accordance with Fitts's law, as the distance of each target from the starting point increased, there should have been a corresponding increase in movement time (MT). However, Adam et al. observed a violation of Fitts's law in the placeholder condition: MTs were not longer to the farthest target than to the second-farthest target, but instead they tended to be shorter. In other words, Adam et al. found that facilitation occurred in the MTs of both horizontal and vertical reaching movements that participants made to the farthest placeholder.

In a subsequent study, Pratt et al. (2007) replicated the effect on MT and extended the finding by manipulating the relative position of the target within the placeholder array independently of the absolute location of the target. That is, they had participants make reaching movements to one of five possible target locations in the presence of three placeholders that could surround the first three, middle three, or final three locations. For example, the three placeholder

Correspondence address: Naseem Al-Aidroos, Department of Psychology, University of Toronto, 100 St. George Street, Toronto, Ontario M5S 3G3, Canada. E-mail address: naseem .al.aidroos@utoronto.ca 


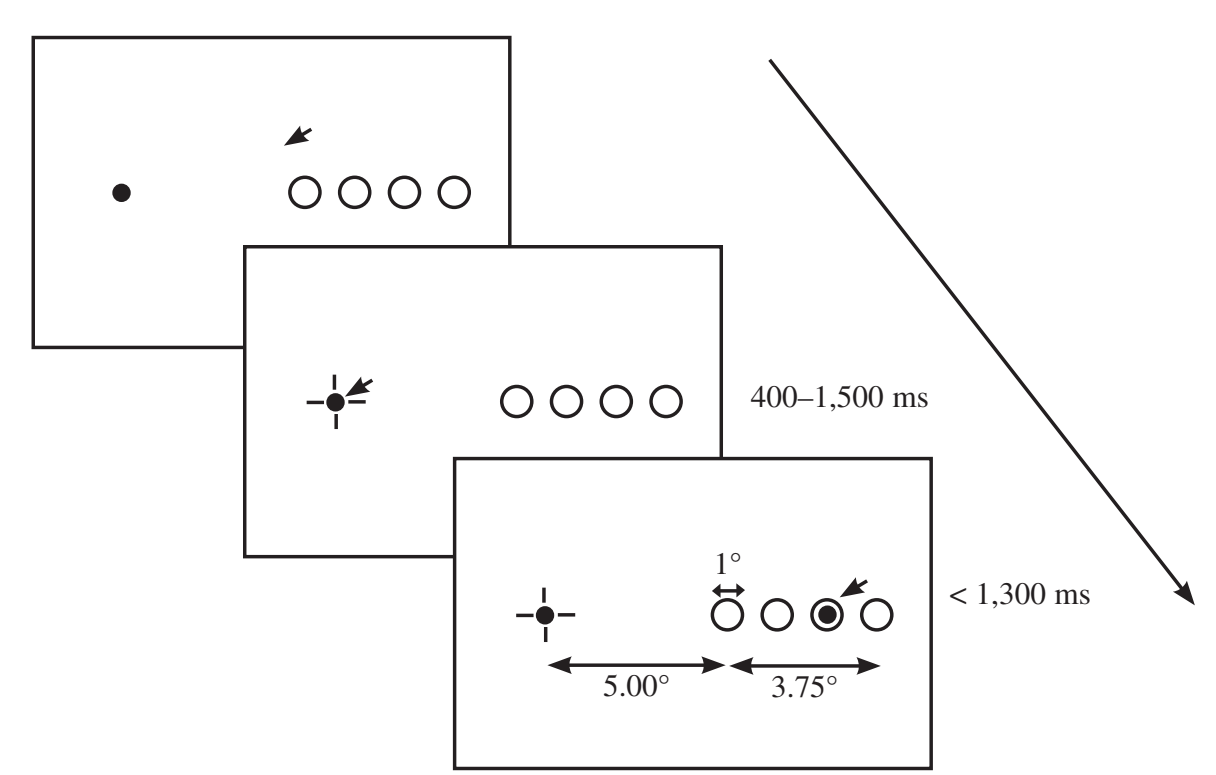

FIGURE 1. Typical sequence of events for a trial in the placeholder condition. The four open circles represent the placeholders that marked the potential target locations, and the black arrow reflects potential gaze position. Placeholders were $1^{\circ}$ wide and were located $5.00^{\circ}, 6.25^{\circ}, 7.50^{\circ}$, and $8.75^{\circ}$ directly to the right of the fixation stimulus. See Design and Procedure for further details.

arrangements allowed movements that were made to the middle of the five target locations to be directed toward the farthest, middle, and nearest placeholder, respectively. As before, MTs to targets at the nearest and farthest placeholders were shorter than were those to the middle placeholder. This manipulation of position within the placeholder array independently of absolute location provided a more powerful test of the effect of placeholders and revealed facilitation not only for movements to the final placeholder but also for movements to the first placeholder.

The finding that movements are facilitated when they are directed toward either the nearest or farthest positions in an array of placeholders complements a secondary result noted by Adam et al. (2006) in their original study. In addition to the aforementioned effect on MT, Adam et al. observed that placeholders attenuated the range effect: The typical overshoots for shorter movements and undershoots for longer movements that characterize the range effect (Poulton, 1974; Searle \& Taylor, 1948) were diminished in the presence of placeholders. Thus, in combination with the effect on MT, visual placeholders caused both faster and more accurate movements to the nearest and farthest positions in the array.

To account for this effect of placeholders, Pratt et al. (2007; see also Adam et al., 2006) proposed a visuomotor explanation. They argued in that proposal that the perceptual array of placeholders forms an allocentric frame of reference that facilitates the spatial processing of the ends of the array (e.g., Adam, Hommel, \& Umiltà, 2003; Mewhort \& Campbell, 1978) and, in turn, movements made to those locations. In particular, Pratt et al. proposed that placehold- ers may make movements to end locations easier to control, thus increasing the speed, or reducing the number, of corrective submovements and MT. It should be noted, however, that the effect on the nearest location may only be revealed in paradigms that control for target amplitude (as Pratt et al. did in their study).

In the present study, we sought to determine if the beneficial effects of placeholders on visuomotor performance of manual movements could be extended to the oculomotor system-in particular, to saccadic eye movements. Saccades are rapid, ballistic eye movements that individuals use to gather visual information from the environment. Because of their unique role in the acquisition of information, saccades are humans' most commonly made overt movement because they make approximately three saccades every second of their waking day. Thus, determining whether placeholders also affect saccades would provide information about both the control mechanisms of the oculomotor system and how such mechanisms affect the acquisition of visual information.

There are reasons to believe that saccades should show effects similar to those of targets in structured perceptual arrays. Performance of saccades, like performance of manual movements, is constrained by both the range effect (Kapoula, 1985) and the speed-accuracy trade-off described in Fitts's law (e.g., Abrams, Meyer, \& Kornblum, 1989; but see Chi \& Lin, 1997). There are, however, a number of reasons to suggest that the effect of placeholders may not occur for saccadic eye movements because not all effects on the performance of manual movements are seen in saccades. For instance, there is a one-target advantage for manual movements (e.g., Adam 
et al., 2000; Glencross, 1980) but not for saccades (Pratt, Shen, \& Adam, 2004). Furthermore, the oculomotor system primarily uses saccades to rapidly gain information from the visual field, whereas it typically uses rapid hand responses to grasp or reach already viewed objects in the environment. In addition, vision is the primary form of sensory feedback in a rapid aiming task. For saccades, however, the eye serves as both the primary source of sensory feedback and the effector. For these reasons, the placeholder effect may not occur for saccadic eye movements. To resolve this issue, in the present study we tested for the effect in the oculomotor system by having participants make saccades in the presence and absence of placeholders.

In addition to accomplishing this primary goal, a test for an effect of placeholders on saccadic eye movements would enable us to refine the visuomotor proposal of Pratt et al. (2007). This refinement is possible because of a difference between the control of manual and saccadic movements. Whereas each discrete manual movement is composed of an initial ballistic phase followed by a corrective-submovement phase (e.g., Meyer, Abrams, Kornblum, Wright, \& Smith, 1988; Woodworth, 1899), a saccade contains only the ballistic phase, with corrections made through additional, secondary saccades (Becker, 1972; Becker \& Fuchs, 1969; Hallett, 1978; Prablanc \& Jeannerod, 1975; Weber \& Daroff, 1972). For this reason, if one observes the placeholder effect for saccadic eye movements, then the effect must not be limited to the corrective-submovement phase.

If one finds an effect of placeholders on the speed or accuracy of saccades, then the effect would have had to arise either in the planning or the execution of the ballistic phase. Changes in the speed or accuracy of a movement are normally achieved by a reorganization of the kinematics of the movement (MacKenzie, Marteniuk, Dugas, Liske, \& Eickmeier, 1987; Mottet \& Bootsma, 1999), which is implemented through changes to a neuromotor control signal (Abrams et al., 1989; cf. Carlton \& Newell, 1988; Newell \& Carlton, 1988). In fact, investigators have proposed that the control signal causes speed-accuracy tradeoffs (Harris \& Wolpert, 1998). According to Harris and Wolpert, the neuromotor control signal contains increasing noise with increases in signal magnitude (see also Clamann, 1969; Matthews, 1996). Thus, for example, when magnification of the control signal increases the speed or amplitude of a movement, movement accuracy decreases because of the increase in signal noise. Therefore, if the effect of placeholders is to alter the execution of saccadic movements, then an alteration of the neuromotor control signal should occur and be revealed in the kinematics of the movements. Alternatively, the effect of placeholders may arise during the programming of the movement, in which case the effect should be observed not in the control signal but in the reaction time of the movement. Thus, we can use the results of the present study to refine the visuomotor proposal of Pratt et al. (2007) by constraining the locus of the placeholder effect to particular phases of movement.
For the purpose of investigating the effect of placeholders on the neuromotor control signal, two kinematic measures are of particular interest: the time for the eye to reach peak velocity and the maximum acceleration the eye achieves. Because of the simple mechanics that generate saccadesin particular, short horizontal saccades - those two measures are thought to directly reflect the control signal that the visuomotor system uses to drive the movement (Abrams et al., 1989; Robinson, 1981). In particular, the visuomotor system initates short saccades $\left(<15^{\circ}\right)$ through a single contraction of agonist muscles with simultaneous suppression of antagonist activity (Fuchs \& Luschei, 1970; Miller, 1958; Robinson, 1970) and does not rely on an antagonist contraction to bring the eye to rest. Instead, friction and elasticity cause the eye to come to rest (Robinson, 1964). Furthermore, the visuomotor system generates horizontal eye movements by using only a single set of muscles (Childress \& Jones, 1967). Thus, the forces that generate a short, horizontal saccade result from a single muscle contraction, and researchers can directly measure the duration and magnitude of the control signal that initiates the contraction from the kinematics of the resulting eye movement (Reinhart \& Zuber, 1971; Thomas, 1969). Because the eye will begin to decelerate once the muscle contraction ends, the saccade's time to peak velocity provides an indication of the control signal's duration. Furthermore, because the acceleration of the eye reflects the magnitude of the force applied to the eye, peak acceleration provides an indication of the magnitude of the control signal. Any effect of placeholders on the duration or magnitude of the neuromotor control signal should therefore be revealed in time to peak velocity and peak acceleration of the saccades, respectively.

To examine whether placeholders have an effect on saccadic eye movements in the present study, we adapted the manual response paradigm of Adam et al. (2006). Participants in the present study made horizontal saccades to suddenly appearing targets at various distances, either in the presence or absence of placeholders. This paradigm enabled us to determine whether the relationship between speed and accuracy was altered by the presence of placeholders. We also examined possible attenuations of the range effect. Because target size does not effectively control the accuracy of saccadic eye movements (as noted by Chi \& Lin, 1997), we instead measured accuracy on the basis of the saccades' end-location variability, with greater variability indicating less accuracy. This procedure accords with Welford, Norris, and Shock's (1969) description of effective target width. Fitts's law predicts that in this paradigm, increases in target distance result in increases in MT or decreases in accuracy, or both. If, however, placeholders have the same effect on saccadic eye movements as they have on manual pointing movements, then saccades to the farthest target position may be completed more quickly or more accurately in the placeholder condition than in the no-placeholder condition. Thus, the following predictions can be made. (a) If the effect of placeholders on the skeletal and ocular motor 
systems is the same, then one should find a decrease in MT or endpoint variability, or both, for saccades made to the farthest target location in the placeholder condition in comparison with those made in the no-placeholder condition. (b) A corresponding attenuation of the range effect for the placeholder condition should also occur. (c) Furthermore, if such an effect on speed or accuracy results from a change in the neuromotor control signal, then one should find a facilitatory effect on peak acceleration or time to peak velocity of the saccade in the placeholder condition in comparison with that in the no-placeholder condition.

\section{Method}

\section{Participants}

Participants were 18 undergraduate students who were enrolled in an introductory psychology course at the University of Toronto and author N.A. As compensation for participating, the undergraduates received extra credit toward their final course grade. All participants had normal or corrected-to-normal vision. We excluded the data of 1 participant from the analyses because of an error in data recording. We received Institutional Review Board approval for our experimental protocol. We carried out the present research in accordance with the Helsinki Declaration.

\section{Apparatus}

We recorded eye movements by monitoring retinal position and corneal reflectance by using a camera-based eye tracker (Eyelink II; SR Research, Mississauga, Ontario, Canada) that had a temporal resolution of $250 \mathrm{~Hz}$ and a spatial resolution of $0.2^{\circ}$. We established gaze position by using a nine-point calibration and validation. We recorded eye position data from whichever eye the Eyelink II tracked most accurately, as determined by the validation procedure. We determined the beginning and end of saccadic eye movements by using a $30 \%$ s threshold, with the additional criterion that the eye should exceed an acceleration of $8,000 \% \mathrm{~s}^{2}$ during the movement. Experimental displays were presented on a $48-\mathrm{cm}$ flat CRT at a refresh rate of 100 $\mathrm{Hz}$ and a resolution of $1024 \times 768$ pixels. We used a chin rest to fix the participant's head $60 \mathrm{~cm}$ from the monitor.

\section{Design and Procedure}

We began each experimental session by verbally instructing participants that they would be required to make rightward eye movements from a fixation stimulus to a target that would appear suddenly in one of four locations. Each participant was further instructed that we would present placeholders to mark the potential target locations for half of the experiment and that we would not present such placeholders for the other half. We counterbalanced the order of those two halves between participants. Thus, the experimental design consisted of (a) one betweenparticipant factor, order of counterbalancing (order), and (b) two within-participant factors, whether placeholders were presented (placeholder, no placeholder) and which of four target positions was presented (target position). After the verbal instructions, we completed the setup of the eye tracker. During setup, the participant performed a nine-point calibration and validation repeatedly until he or she attained a minimum average accuracy of $0.5^{\circ}$. Each participant performed 136 trials (16 practice trials plus three blocks of 40 experimental trials) twice, once for each placeholder condition. On any trial, or between any blocks of trials, the experimenter could elect to perform a drift correction or recalibration of the eye tracker.

We presented all stimuli on a black background. The initial display on every trial contained a fixation stimulus: a green circle with a diameter subtending $0.3^{\circ}$ of visual angle, centered vertically on the display but offset $5^{\circ}$ to the left of the horizontal center (see Figure 1). On placeholder trials, we also presented placeholders that demarcated the four potential target positions located $5.00^{\circ}, 6.25^{\circ}, 7.50^{\circ}$, and $8.75^{\circ}$ directly to the right of the fixation stimulus (we measured distances center to center). We presented placeholders as gray rings with an outer diameter of $1.00^{\circ}$ and an inner diameter of $0.95^{\circ}$.

The trial began once participants moved their gaze to within $1^{\circ}$ of the fixation stimulus. To inform participants that a trial had begun, we presented a crosshair around the fixation stimulus. Once the trial commenced, we gave participants a 1,500-ms interval to establish and maintain fixation for at least $400 \mathrm{~ms}$, at which point a target was presented randomly in one of the four target positions. Targets were identical in appearance to the fixation stimulus. Following target presentation, we required participants to initiate a first saccade of at least $3.15^{\circ}$ within $1,300 \mathrm{~ms}$. If participants executed such a saccade successfully, after a delay of $150 \mathrm{~ms}$, a $1000-\mathrm{Hz}$ success tone was sounded for $100 \mathrm{~ms}$, and the stimuli on the display were then extinguished. If participants were unable to initially maintain fixation or to subsequently generate the defined saccade, however, a 200-Hz error tone was sounded for $100 \mathrm{~ms}$, and the stimuli on the display were then extinguished. The display then remained blank for $800 \mathrm{~ms}$, after which the next trial began.

\section{Measures}

We used six measures to evaluate the effect of placeholders and target positions on saccadic movements: (a) To evaluate motor planning, we determined saccadic reaction time (RT), the time between target onset and initiation of the saccadic response. (b) To evaluate the speed of saccades, we used saccadic MT, the time between the initiation and the completion of the first saccade. (c) To evaluate accuracy, we used the standard deviation of the horizontal endpoints of the saccades. (d) We calculated saccadic velocity on the basis of the distance that the eye traveled during each sample of the recorded eye movements, and we recorded the time (from saccade onset) to peak velocity to evaluate the duration of the neuromotor control signal. (e) We calculated saccadic acceleration on the basis of the change in velocity 
between recorded samples, and we used peak acceleration to evaluate the magnitude of the control signal. (f) We used constant error to evaluate the range effect. Specifically, we recorded the on-screen position of gaze by using a Cartesian coordinate system, and the signed difference between the horizontal coordinate at the completion of a saccade and the center of the presented target provided the measure of constant error.

\section{Data Analysis}

Before analyzing saccadic measures, we excluded error trials from the dataset. Error trials included trials for which (a) the participant was unable to properly establish fixation at the beginning of the trial, (b) RT was less than 100 ms or greater than $400 \mathrm{~ms}$, (c) MT was less than $10 \mathrm{~ms}$ or greater than $100 \mathrm{~ms}$, (d) the initial saccade did not exceed an amplitude of $3.15^{\circ}$, or (e) the initial saccade did not land within $2^{\circ}$ of the target.

To evaluate the effect of placeholders on each saccadic measure, we performed separate 2 (order) $\times 2$ (placeholder) $\times$ 4 (target position) mixed analyses of variance (ANOVAs). No main effects of the order of counterbalancing were observed. However, order did interact with placeholder and target position for the constant error measure. For this reason, the analysis of constant error, which we present at the end of the Results section, includes separate analyses for each counterbalancing order. For the remaining measures, however, we collapsed the analyses across order of counterbalancing and performed the analyses by using 2 (placeholder) $\times 4$ (target position) within-participant ANOVAs. In addition, we followed each ANOVA with a planned two-tailed $t$ test to compare the effect of placeholders on saccades made to the farthest target location. We adjusted degrees of freedom by using the Greenhouse-Geisser correction in all analyses in which the assumption of sphericity was not justified, as indicated by Mauchly's test of sphericity.

\section{Results}

Removal of error trials resulted in elimination of $22.5 \%$ of the trials, the majority $(80.1 \%)$ from fixation errors. A 2 (order) $\times 2$ (placeholder) $\times 4$ (target position) mixed ANOVA revealed that the number of errors did not differ significantly between conditions (all $F$ s $<1.2$ ).

\section{Reaction Time}

We performed a 2 (placeholder) $\times 4$ (target position) within-participant ANOVA on RT. This analysis revealed no main effect of placeholder, $F(1,18)=2.17, p=.158$ $(M S E=1,095.80)$, no main effect of target position, $F(1.7$, $31.1)=2.01, p=.156(M S E=302.19)$, and no interaction, $F(3,54)=1.22, p=.310(M S E=168.41)$. The planned $t$ test, however, did reveal a significant difference, $t(18)=$ 2.30, $p=.034$. As can be seen in Table 1, participants' RTs for saccades made toward the farthest target position were significantly slower in the presence of placeholders than in their absence. The increase in RT suggests that additional motor planning occurred for saccades generated toward the farthest target position in the placeholder condition.

\section{MT}

To begin the evaluation of the relationship between speed and accuracy, we investigated the effect of placeholder and target position conditions on MTs by conducting a 2 (placeholder) $\times 4$ (target position) within-participant ANOVA. The analysis revealed a strong effect of target position, $F(2.0,35.5)=$ $545.49, p<.0001, \eta^{2}=.97(M S E=1.83)$, but no effect of

\section{TABLE 1. Saccadic Measurements as a Function of Placeholder Condition and Target Distance}

\begin{tabular}{|c|c|c|c|c|}
\hline \multirow[b]{2}{*}{ Condition } & \multicolumn{4}{|c|}{ Target distance } \\
\hline & $5.00^{\circ}$ & $6.25^{\circ}$ & $7.50^{\circ}$ & $8.75^{\circ}$ \\
\hline \multicolumn{5}{|c|}{ Reaction time $(\mathrm{ms})$} \\
\hline No-placeholder & 224 & 219 & 216 & 218 \\
\hline Placeholder & 228 & 219 & 224 & 227 \\
\hline Difference & -3.5 & 0.2 & -8.1 & $-10.1^{*}$ \\
\hline \multicolumn{5}{|c|}{ Peak acceleration $\left(\% s^{2}\right)$} \\
\hline No-placeholder & 28,760 & 31,407 & 32,398 & 34,409 \\
\hline Placeholder & 29,916 & 31,685 & 33,794 & 36,405 \\
\hline Difference & $-1,156$ & -278 & $-1,396$ & $-1,995$ \\
\hline \multicolumn{5}{|c|}{ Time to peak velocity $(\mathrm{ms})$} \\
\hline No-placeholder & 20.5 & 21.6 & 23.3 & 24.7 \\
\hline Placeholder & 20.3 & 21.4 & 23.1 & 24.2 \\
\hline Difference & 0.14 & 0.16 & 0.13 & 0.55 \\
\hline
\end{tabular}


placeholder and no interaction, both $F_{\mathrm{S}}<1.1$. The planned $t$ test comparing MTs toward the farthest target in the placeholder and no-placeholder conditions was not significant, $t(18)=1.10, p>.28$. As can be seen in Figure 2, these results revealed that movement amplitude almost entirely determined MT; more distant movements took longer to complete. To further investigate the relationship between distance and speed, we regressed mean MT onto mean movement amplitude for each of the four target positions in both placeholder conditions. The regression analysis revealed a strong linear relationship between saccadic amplitude and saccadic MT both when placeholders were present, $b=2.67, t(2)=25.14, p=.002$, and when they were absent, $b=2.60, t(2)=74.88, p<.001$. The slopes $b$ of the analyses did not differ, $t(4)=0.49, p>.5$. Thus, although researchers found an effect of placeholders on MTs of manual movements in previous studies, we did not find faster MTs for saccadic movements to the farthest target position in the placeholder condition $(M=42.30, S D=3.33)$ than in the no-placeholder condition $(M=42.95, S D=3.53)$ in the present study. Instead, movement amplitude almost entirely determined saccadic MTs.

\section{Endpoint Variability}

To evaluate the effect of placeholders and target positions on accuracy, we performed a 2 (placeholder) $\times 4$ (target position) within-participant ANOVA on horizontal endpoint variability. The analysis revealed a main effect of target position, $F(3,54)=4.13, p=.010(M S E=0.01)$, and a significant two-way interaction between target position and placeholder, $F(3,54)=4.32, p=.008(M S E=0.01)$. The main effect of placeholder was not significant, $F<1$. As can be seen in Figure 2, saccadic endpoint variability tended to increase with amplitude. However, the presence of placeholders altered that relationship for the farthest target position. There was less variability in the placeholder condition $(M=0.53, S D=0.12)$ than in the no-placeholder condition $(M=0.62, S D=0.17)$. Most important, the planned $t$ test for the farthest target position revealed that the reduction in endpoint variability in the placeholder condition was significant, $t(18)=2.57, p=.019$.

\section{Peak Acceleration and Time to Peak Velocity}

To investigate whether the placeholder and target position conditions affected the neuromotor control signal that participants used to generate the saccade, we performed a 2 (placeholder) $\times 4$ (target position) within-participant ANOVA on the peak acceleration and time to peak velocity measures. As can be seen in Table 1, placeholders had no reliable effects on either measure. Beginning first with peak acceleration, the analysis revealed a strong effect

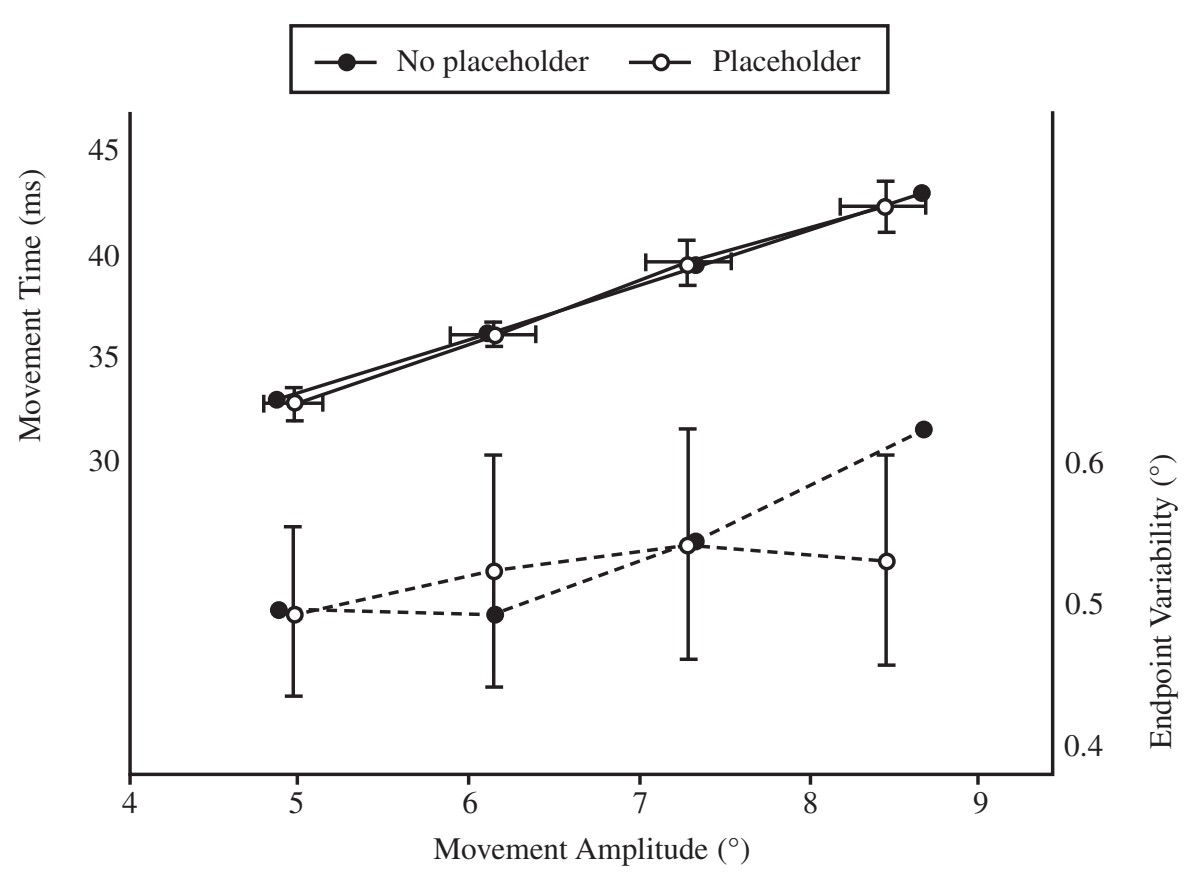

FIGURE 2. Movement time (upper plot) and standard deviation of horizontal endpoints (endpoint variability; lower plot) as a function of target position and placeholder condition. Both metrics are plotted against actual movement amplitude. The nearest-farthest target positions on any line can be read from left to right. Three results can be observed. (a) Movement amplitude was largely determined by target position and was unaffected by placeholder presence. (b) MT was similarly unaffected by placeholders. (c) Placeholders affected movement accuracy, resulting in less variable movements to the farthest target location. Error bars represent $95 \%$ confidence intervals on the differences between means (placeholder vs. no placeholder). 
of target position, $F(1.6,28.0)=30.53, p<.0001, \eta^{2}=$ $.63\left(M S E=15.72 \times 10^{5}\right)$, but no effect of placeholder and no interaction between the two factors, both $F \mathrm{~s}<1$. As target amplitude increased, greater peak accelerations were reached. However, the planned $t$ test revealed that there was no significant effect of placeholders on peak acceleration to the farthest target position, $t(18)=0.97$, $p=.347$. This finding suggests that the magnitude of the neuromotor control signal was unaffected by the presence of placeholders.

The same analysis on time to peak velocity once again revealed a strong effect of target position, $F(1.6,28.2)=89.27$, $p<.0001, \eta^{2}=.83(M S E=2.66)$, but no effect of placeholder, $F(1,18)=1.96, p=.178(M S E=1.17)$, and no interaction between the two factors, $F<1$. As we observed for peak acceleration, time to peak velocity was unaffected by the presence of placeholders, which suggests that placeholders have no effect on the duration of the neuromotor control signal. In particular, the difference between placeholder conditions for the farthest target location was not significant, $t(18)<1.4, p>.20$. Thus, the relationship between saccade amplitude and both peak acceleration and time to peak velocity was unaffected by the presence of placeholders, suggesting that one cannot attribute the detected decrease in endpoint variability to a change in the magnitude or duration of the control signal.

\section{Constant Error}

Last, we performed a 2 (order) $\times 2$ (placeholder) $\times 4$ (target position) mixed ANOVA on constant error. The analysis revealed a significant three-way interaction, $F(3$, $51)=5.72, p=.002(M S E=0.08)$, indicating that the effect of placeholders on the range effect depended on the order of counterbalancing. Thus, we performed a 2 (placeholder) $\times$ 4 (target position) within-participant ANOVA for those participants who completed the no-placeholder condition first. This analysis revealed no main effect of placeholder, $F<1$; no main effect of target position, $F<1$; and no interaction, $F(3,27)=1.47, p=.244\left(M S E=8.48 \times 10^{-3}\right)$. As can be seen in the left panel of Figure 3, there was no range effect in either placeholder condition when the no-placeholder condition was performed first. We also performed the same two-factor ANOVA on those participants who completed the placeholder condition first. As might be expected from the right panel of Figure 3, there was a reliable range effect when placeholders were present (i.e., overshoots to the nearest target and undershoots to the farthest) but no range effect when placeholders were absent. This pattern was revealed by a main effect of target position, $F(3,24)=$ $6.18, p=.003(M S E=0.12)$, and a significant Placeholder $\times$ Target Position interaction, $F(3,24)=10.47, p<.0001$ $(M S E=0.16)$, with no main effect of placeholder, $F<1$.

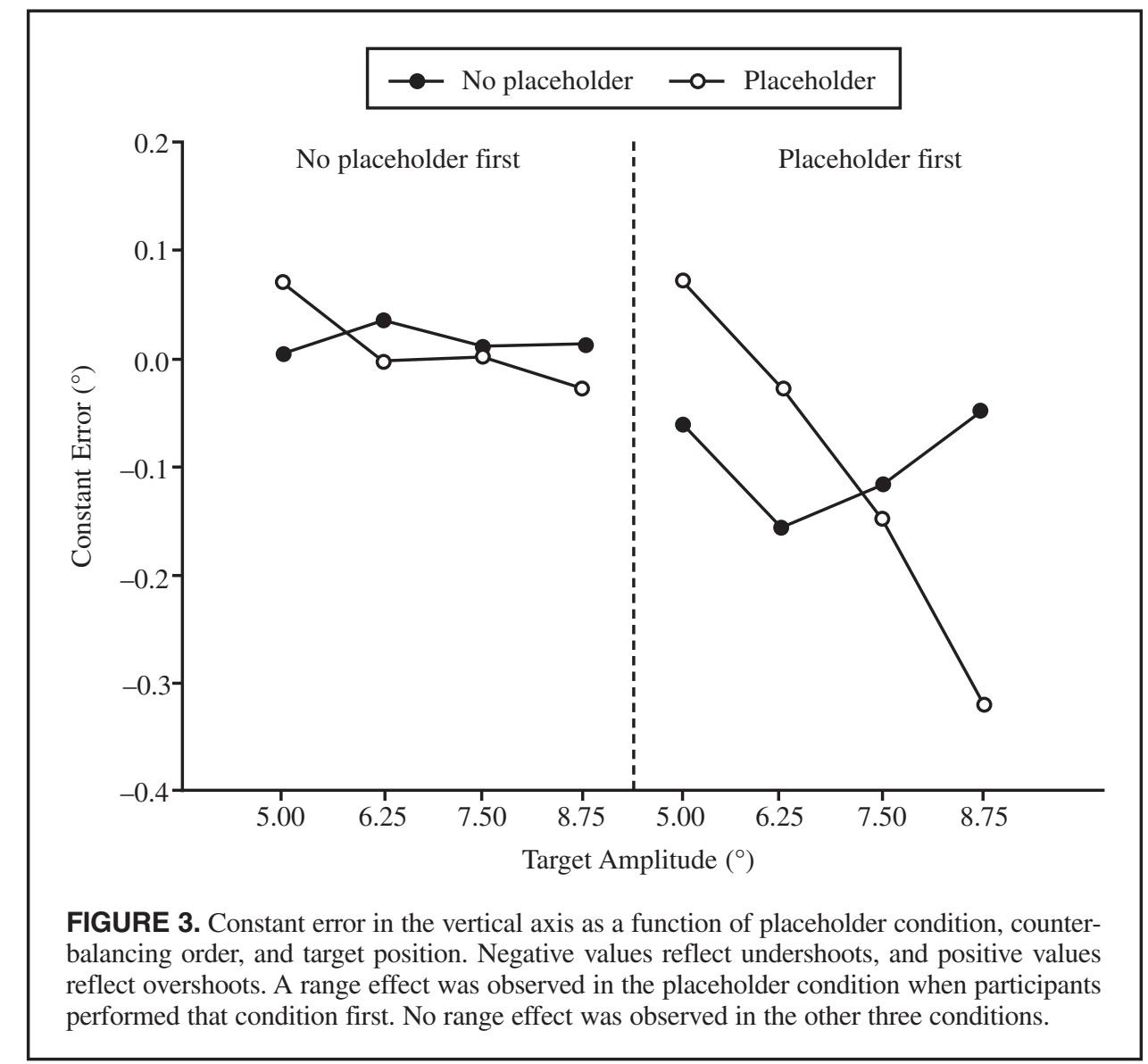

March 2008, Vol. 40, No. 2 
Furthermore, the simple effect of target position for each level of placeholder was significant in the placeholder condition, $F(3,24)=11.42, p<.001(M S E=0.26)$, but not in the no-placeholder condition, $F(3,24)=1.77, p=.177$ $(M S E=0.02)$. The enhanced range effect in the presence of placeholders is contrary to the pattern that Adam et al. (2006) found for manual movements, in which the presence of placeholders reduced the range effect.

\section{Discussion}

Our purpose in the present study was to investigate the effect of a structured perceptual array (in this case, a set of placeholders) on the relationship between the speed and accuracy of saccadic eye movements. It is notable that we found an alteration of the relationship between speed and accuracy, similar to that found for manual movements. However, the alteration was expressed in accuracy rather than speed. That is, placeholders caused an increase in the accuracy of eye movements to the farthest target position via reduced endpoint variability without causing a decrease in speed. Thus, the results of the present study showed the oculomotor equivalent of the placeholder effect that (Adam et al. (2006) and Pratt et al. (2007) have previously found with manual pointing responses. It is important to note that the stimulus displays that we used in the present research were very similar to those that were used previously. For example, the movement difficulties (which Fitts's law quantifies as the ratio between movement amplitude and target width) were equivalent in this study to those in the manual response studies. Thus, it is unlikely that the change from an effect on speed to an effect on accuracy was a result of the particular paradigm we used. Rather, the change was probably a specific result of the switch from manual movements to saccades. Regardless of how the change was expressed, however, the results of the present study clearly demonstrate that the beneficial effect of placeholders extends to the oculomotor system.

The finding that the placeholder effect occurs in the oculomotor system as a change in accuracy rather than speed provides interesting implications for the visuomotor proposal of Pratt et al. (2007). Pratt et al. argued that placeholders facilitate the spatial processing of the ends of the visual array, in turn speeding the corrective phase of movements directed toward the end locations. In contrast, the results of the present study point to an early effect of placeholders. That the RT was slowed for movements to the farthest location in the placeholder condition suggests that participants' visuomotor systems spent additional time programming the saccadic movement. Furthermore, the analysis of saccade dynamics shows that the increase in accuracy did not result from a kinematic reorganization (MacKenzie et al., 1987). In fact, there was no evidence of a change in the duration or magnitude of the underlying neuromotor control signal. Therefore, placeholders appear to affect the programming, rather than the execution, of saccadic eye movements. Although such a conclusion does not contradict the Pratt et al. visuomotor proposal, it does suggest a refinement of the proposal.

Before refining the proposal, however, it is worthwhile to consider why the placeholder effect may be expressed in MT for manual movements but in accuracy for saccadic eye movements. It is notable that given the opportunity, people tend to trade any increase in accuracy for an increase in speed. According to a number of successful models of movement trajectories of both manual and saccadic eye movements, the motor system selects movement speed to attain a criterion level of accuracy (Harris \& Wolpert, 1998, 2006; Tanaka, Krakauer, \& Qian, 2006). Thus, it should not be surprising if an initial increase in the accuracy of manual movements was traded for an increase in speed.

Furthermore, there are two ways in which manual movements may be speeded (at the cost of accuracy). Both the ballistic phase and the corrective-submovement phase might be speeded. However, it may not be possible for either of these two phases to be speeded in saccadic eye movements. As discussed earlier, there is no corrective submovement phase in saccades. Instead, saccades consist of only an initial ballistic phase, with corrections made through the generation of additional, secondary saccades (e.g., Becker, 1972; Prablanc $\&$ Jeannerod, 1975). Thus, the corrective-submovement phase is not available for producing faster saccades. In addition, it may not be possible to speed the ballistic phase of saccades. In particular, saccades made under a considerable variety of situations all follow the main sequence of normal saccades (Bahill, Clark, \& Stark, 1975), which describes a specific relationship between saccade amplitude, duration, and peak velocity. For these reasons, the facilitatory effect of placeholders on manual movements may be exhibited as an increase in movement speed, whereas the same facilitation may be manifested only as an increase in accuracy in the case of saccadic eye movements.

Because of this characterization of the effect of placeholders, the results of the present study suggest that the visuomotor proposal should be refined as follows. In accordance with the proposal of Pratt et al. (2007), the effect of placeholders may be to enhance the spatial processing of the end locations of the visual array. In contrast to the original proposal, however, the present results point to facilitation of the programming of the ballistic phase-which results in a more accurate ballistic movement-rather than facilitation of corrective submovements. For manual movements, the initial increase in accuracy may later be traded for a faster ballistic movement. Alternatively, given a more accurate ballistic phase, the motor system may require fewer submovements to reach the target, again resulting in a decrease in MT. Although the present results do not preclude the possibility that placeholders also directly facilitate the corrective-submovement phase of manual movements, they do demonstrate that the effect cannot be limited to that phase. Indeed, if placeholders facilitate spatial processing, then one would expect the effect to also be beneficial for the implementation of corrective submovements. 
There are, however, some issues regarding the effects of placeholders on the two systems that researchers must account for before they can fully adopt this refined proposal. For example, previous investigators have found RT to be unrelated to the facilitatory effect of placeholders on manual movements (Adam et al., 2006; Pratt et al., 2007), whereas we found longer RTs when placeholders increased accuracy in the present study. In addition, the results of the range-effect analysis point to further differences between the effect of placeholders on the manual and oculomotor systems. Although a range effect is typically exhibited by both systems, a greater range effect for manual movements was previously found (Adam et al.) in the no-placeholder condition. In the present study, however, we found a greater effect in the placeholder condition for saccadic eye movements (but only when we presented the placeholder condition first). It is possible that the observed range effect was actually a result of the global effect (or saccadic averaging effect) - the tendency for stimulus-driven saccades to be drawn to roughly the center of gravity of all visible stimuli located near the target (Coren \& Hoenig, 1972). Thus, in the placeholder condition, saccades may have been drawn to the center of the perceptual array, generating a range effect. It is curious that the range effect was present only when participants performed the placeholder condition first. However, this finding may indicate that the global effect is not as inflexible as previously thought (Findlay, 1982).

At present, the results of the range effect and RT analyses suggest that there are subtle differences in the general effects of placeholders on manual movements and saccadic eye movements. Furthermore, researchers do not currently know how the programmed and corrective portions of manual movements differ in the presence and absence of placeholders. However, experiments are currently underway in our laboratory that will help elucidate the fine details regarding the planning and production of responses to targets in structured perceptual arrays.

What clearly emerges from this study is that placeholders affect the manual and oculomotor systems, as exhibited by facilitation of both types of movements to the farthest target location. Thus, our results demonstrate that the modulation of Fitts's law holds for both manual and oculomotor movements and, further, that the effect may arise early in the programming of the movement. Because of their different time courses and control modes, however, the tradeoff is expressed differently for the two motor systems (Harris \& Wolpert, 1998): Facilitation is exhibited in manual movements as an increase in speed and in saccades as an increase in accuracy.

\section{ACKNOWLEDGMENTS}

A Natural Sciences and Engineering Research Council of Canada (NSERC) Discovery Grant to Jay Pratt and a NSERC Canadian Graduate Scholarship to Naseem Al-Aidroos supported this research.

\section{Biographical Information}

Naseem Al-Aidroos does not teach; his research interests include visual cognition, attention, perception-action coupling, and consciousness.
Martin H. Fischer teaches courses on motor control. His research interests include numerical cognition, in particular the spaital numerical association of response code (SNARC) effect, spatial memory in reading, joint attention (with posture cues and gaze cues), and various aspects of motor cognition.

Jos J. Adam teaches courses on motor control. His research interests include visuomotor attention, control of hand movements, perception-action coupling, and their changes with aging.

Jay Pratt teaches courses on attention and performance. His research interests include visual cognition, attention, eye movements, motor control, and their changes with aging.

\section{REFERENCES}

Abrams, R. A., Meyer, D. E., \& Kornblum, S. (1989). Speed and accuracy of saccadic eye movements: Characteristics of impulse variability in the oculomotor system. Journal of Experimental Psychology: Human Perception and Performance, 15, 529-543.

Adam, J. J., Hommel, B., \& Umiltà, C. (2003). Preparing for perception and action: I. The role of grouping in the responsecueing paradigm. Cognitive Psychology, 46, 302-358.

Adam, J. J., Mol, R., Pratt, J., \& Fischer, M. H. (2006). Moving farther but faster: An exception to Fitts's law. Psychological Science, 17, 794-798.

Adam, J. J., Nieuwenstein, J., Huys, R., Paas, F., Kingma, H., Willems, P., et al. (2000). Control of rapid aimed hand movements: The one-target advantage. Journal of Experimental Psychology: Human Perception and Performance, 26, 295-312.

Bahill, A. T., Clark, M. R., \& Stark, L. (1975). The main sequence: A tool for studying human eye movements. Mathematical Biosciences, 24, 191-204.

Becker, W. (1972). The control of eye movements in the saccadic system. Bibliotheca Ophthalmologica, 82, 233-243.

Becker, W., \& Fuchs, A. F. (1969). Further properties of the human saccadic system: Eye movements and correction saccades with and without visual fixation points. Vision Research, 9, 1247-1258.

Carlton, L. G., \& Newell, K. M. (1988). Force variability and movement accuracy in space-time. Journal of Experimental Psychology: Human Perception and Performance, 14, 24-36.

Chi, C.-F., \& Lin, C.-L. (1997). Speed and accuracy of eye-gaze pointing. Perceptual \& Motor Skills, 85, 705-718.

Childress, D. S., \& Jones, R. W. (1967). Mechanics of horizontal movement of the human eye. The Journal of Physiology, 188, 273-284.

Clamann, H. P. (1969). Statistical analysis of motor unit firing patterns in a human skeletal muscle. Biophysical Journal, 9, $1233-1251$.

Coren, S., \& Hoenig, P. (1972). Effect of non-target stimuli upon length of voluntary saccades. Perceptual and Motor Skills, 34, 499-508.

Findlay, J. (1982). Global visual processing for saccadic eye movements. Vision Research, 22, 1033-1045.

Fitts, P. M. (1954). The information capacity of the human motor system in controlling the amplitude of movement. Journal of Experimental Psychology, 47, 381-389.

Fitts, P. M., \& Peterson, J. R. (1964). Information capacity of discrete motor responses. Journal of Experimental Psychology, 67, 103-112.

Fuchs, A. F., \& Luschei, E. S. (1970). Firing patterns of abducens neurons of alert monkeys in relationship to horizontal eye movement. Journal of Neurophysiology, 33, 382-392.

Glencross, J. D. (1980). Response planning and the organization of speed movements. In R. S. Nickerson (Ed.), Attention and performance VIII (pp. 107-125). Hillsdale, NJ: Erlbaum.

Hallett, P. E. (1978). Primary and secondary saccades to goals defined by instructions. Vision Research, 18, 1279-1292. 
Harris, C. M., \& Wolpert, D. M. (1998, August 20). Signaldependent noise determines motor planning. Nature, 394(6695), 780-784.

Harris, C. M., \& Wolpert, D. M. (2006). The main sequence of saccades optimizes speed-accuracy trade-off. Biological Cybernetics, 95, 21-29.

Kapoula, Z. (1985). Evidence for a range effect in the saccadic system. Vision Research, 25, 1155-1157.

MacKenzie, C. L., Marteniuk, R. G., Dugas, C., Liske, D., \& Eickmeier, B. (1987). Three-dimensional movement trajectories in Fitts' task: Implications for control. Quarterly Journal of Experimental Psychology, 39A, 629-647.

Matthews, P. B. C. (1996). Relationship of firing intervals of human motor units to the trajectory of post-spike afterhyperpolarization and synaptic noise. The Journal of Physiology, 492, 597-628.

Mewhort, D. J. K., \& Campbell, A. J. (1978). Processing spatial information and the selective-masking effect. Perception \& Psychophysics, 24, 93-101.

Meyer, D. E., Abrams, R. A., Kornblum, S., Wright, C. E., \& Smith, J. E. K. (1988). Optimality in human motor performance: Ideal control of rapid aimed movements. Psychological Review, 95, 340-370.

Miller, J. E. (1958). Electromyographic pattern of saccadic eye movements. American Journal of Ophthalmology, 46, 183-186.

Mottet, D., \& Bootsma, R. J. (1999). The dynamics of goal-directed rhythmical aiming. Biological Cybernetics, 80, 235-245.

Newell, K. M., \& Carlton, L. G. (1988). Force variability in isometric responses. Journal of Experimental Psychology: Human Perception and Performance, 14, 37-44.

Plamondon, R., \& Alimi, A. M. (1997). Speed/accuracy trade-offs in target-directed movements. Behavioral and Brain Sciences, 20, 279-349.

Poulton, E. C. (1974). Tracking skill and manual control. New York: Academic Press.

Prablanc, C., \& Jeannerod, M. (1975). Corrective saccades: Dependence on retinal reafferent signals. Vision Research, 18, 557-560.
Pratt, J., Adam, J. J., \& Fischer, M. H. (2007). Visual layout modulates Fitts's law: The importance of first and last positions. Psychonomic Bulletin \& Review, 14, 350-355.

Pratt, J., Shen, J., \& Adam, J. (2004). The planning and execution of sequential eye movements: Saccades do not show the one-target advantage. Human Movement Science, 22, 679-688.

Reinhart, R. J., \& Zuber, B. L. (1971). Parameters of the control signals for saccadic eye movement: Electrical stimulation and modeling. Experimental Neurology, 30, 148-161.

Robinson, D. A. (1964). The mechanics of human saccadic eye movement. The Journal of Physiology, 174, 245-264.

Robinson, D. A. (1970). Oculomotor unit behavior in the monkey. Journal of Neurophysiology, 33, 393-404.

Robinson, D. A. (1981). The use of control systems analysis in the neurophysiology of eye movements. Annual Review of Neuroscience, 4, 463-503.

Searle, L. V., \& Taylor, F. V. (1948). Studies of tracking behaviour. I. Rate and time characteristics of simple corrective movements. Journal of Experimental Psychology, 38, 615-631.

Tanaka, H., Krakauer, J. W., \& Qian, N. (2006). An optimization principle for determining movement duration. Journal of Neurophysiology, 95, 3875-3886.

Thomas, J. G. (1969). The dynamics of small saccadic eye movements. The Journal of Physiology, 200, 109-127.

Weber, R. B., \& Daroff, R. B. (1972). Corrective movements following refixation saccades: Type and control system analysis. Vision Research, 12, 467-475.

Welford, A. T., Norris, A. H., \& Shock, N. W. (1969). Speed and accuracy of movement and their changes with age. Acta Psychologica, 30, 3-15.

Woodworth, R. S. (1899). The accuracy of voluntary movement. Psychological Monographs, 3, 1-114.

Submitted November 30, 2006

Revised March 30, 2007 
Reproduced with permission of the copyright owner. Further reproduction prohibited without permission. 